\title{
Chiara Torre
}

\section{Seneca vs Seneca: generazioni e stili a confronto tra oratoria, filosofia e storiografia}

\begin{abstract}
This paper aims to offer a fresh reading about Seneca's epistle 100 to Lucilius about the stylistic portrait of Papirius Fabianus. As we know, this portrait is drawn on the sketch that Seneca the father had already traced in the preface to Controversiae book 2. So, epistle 100 too could be read as a little 'literary memoir', just like Seneca the father's prefaces should be interpreted.

Two nodal points will be highlighted in this paper: 1) Seneca evaluates Fabianus' eloquence from a technical point of view, that is his compositio; in so doing, he reuses some critical patterns that Cicero had applied to historical writings, as well as he sets a comparative judgement between philosophy and rhetoric in the same way Seneca the father had set the supremacy of history on the rhetoric. 2) Both the father and the son consider Papirius Fabianus as a sort of a 'cultural icon', useful to represent the crucial and changeable crossing of literary genres in the first imperial Age.
\end{abstract}

\section{Sulle orme del padre (I): l'epistola 100 come literary memoir}

Il titolo del mio contributo allude al confronto implicito (ma non troppo) che Seneca, nella lettera 100 indirizzata a Lucilio, ingaggia a distanza con il padre in merito al giudizio sullo stile del declamatore e filosofo Fabiano, ${ }^{1}$ di cui, come è noto, Seneca padre aveva tracciato un vivido ritratto nella prefazione al secondo libro delle Controversiae. Al centro dell'epistola sta una questione, con una lunga e intricata tradizione alle spalle, che non è storiografica, ma piuttosto retorica e filosofica: quale stile per il filosofo?

\footnotetext{
1 Su Papirio Fabiano rimando al sintetico ma esaustivo Berti (2018) 313-315 (con rassegna bibliografica). Mazzoli (1967) 252-259 colloca il tirocinium di Seneca presso la scuola dei Sestii, dove egli ebbe anche Fabiano tra i suoi maestri, all'inizio della sua formazione filosofica, presumibilmente nel periodo compreso tra il 13 e il 19 d.C. (anno dell'editto di Tiberio contro i culti stranieri, che determinò la chiusura della secta sestiana).
}

D Open Access. (c) 2020 Chiara Torre, published by De Gruyter. (c) BY-NC-ND This work is licensed under the Creative Commons Attribution-NonCommercial-NoDerivatives 4.0 License. https://doi.org/10.1515/9783110688665-015 
In questa sede, però, non è mia intenzione affrontare il problema del rapporto tra filosofia e retorica in relazione alla teoria e alla prassi della parenesi senecana. Piuttosto, vorrei proporre una lettura retrospettiva dell'epist. 100 come documento storico-culturale relativo a quel milieu nel quale si svolse la formazione giovanile di Seneca, fortemente influenzata dall'impronta paterna; e, più specificamente, vorrei provare a evidenziare, nel giudizio sulla compositio di Fabiano, alcuni tratti che potrebbero alludere, tra l'altro, allo stile dello storico, da leggersi in filigrana dietro alle considerazioni sullo stile del filosofo.

Prima, però, di sviluppare il confronto tra l'epistola 100 a Lucilio e la seconda praefatio delle Controversiae è opportuno rimarcare come lo sguardo retrospettivo, che si intende ora adottare, ricalchi in realtà un atteggiamento analogamente retrospettivo di Seneca, che a sua volta lo eredita dalla scrittura di Seneca padre: non già, però, dalla scrittura storiografica di quest'ultimo (l'epistola 100 non è, com'è ovvio, figlia delle Historiae), bensì da quei 'memorial writings', a metà tra autobiografia e critica letteraria, rappresentati appunto dalle praefationes ai libri delle Controversiae (1, 2, 3, 4, 7, 9, 10), che Seneca padre dedica ai figli adottando, per primo tra gli scrittori latini di retorica, proprio la forma epistolare. $^{2}$

Nella prefazione al primo libro, rispondendo a una precisa richiesta dei figli e pur lamentando la fragilità della sua memoria, Seneca afferma di voler riportare per iscritto tanto i ritratti letterari dei declamatori che ha ascoltato in gioventù, quanto le loro parole, in modo che i figli stessi possano preservarne il ricordo ed esercitare il loro personale giudizio critico.

È questa, come mi pare, la traccia del modello della lettera 100. L'analisi dello stile di Fabiano vi è condotta a due voci: da un parte (nell'incipit), la voce di Lucilio, di cui Seneca riferisce alcune perplessità scaturite dalla recente lettura degli scritti di Fabiano; dall'altra (in chiusura), la voce di Seneca stesso, che tenta una rivalutazione dello stile del maestro, basandosi su un ricordo personale, ma ormai datato e dunque sfocato, del suo insegnamento orale. ${ }^{3}$ La memoria autobio-grafica, venata di intima gratitudine per un maestro che suscitava nei suoi giovani allievi non

2 La particolare caratura 'memorialistica' delle praefationes senecane è stata adeguatamente valorizzata da Sussmann (1971) 285-291 e, soprattutto, da Citti (2005) 173-187.

3 Sen. epist. 100.1: Fabiani Papiri libros qui inscribuntur civilium legisse te cupidissime scribis et non respondisse expectationi tuae; epist. 100.12: talia esse scripta eius non dubito, etiam si magis reminiscor quam teneo haeretque mihi color eorum non ex recenti conversatione familiariter sed summatim, ut solet ex vetere notitia; cum audirem certe illum, talia mihi videbantur, non solida sed plena, quae adulescentem indolis bonae attollerent et ad imitationem sui evocarent sine desperatione vincendi, quae mihi adhortatio videtur efficacissima. Deterret enim qui imitandi cupiditatem fecit, spem abstulit. 
solo il desiderio di imitarlo ma anche la fiducia di poterlo superare, viene per così dire riattivata dalle osservazioni critiche di Lucilio sugli scritti di Fabiano e sul suo stile. Si tratta, a ben vedere, della stessa dinamica che innesca la scrittura memoriale di Seneca padre; tale dinamica, e relativo modello, garantiscono perciò all'epistola 100 una posizione assai peculiare all'interno del nutrito gruppo di lettere dedicate, nel medesimo epistolario, a dibattere vari aspetti dello stile in senso generale o in rapporto alla filosofia: lettere che, come è noto, intrattengono una fitta e conclamata serie di rapporti con la nostra epi-stola su Fabiano, ${ }^{4}$ ma la cui facies è in realtà diversa proprio per l'assenza del filtro del ricordo personale e dell'esperienza di scuola.

Viceversa, per questo aspetto 'memoriale', l'epistola 100 va piuttosto accostata all'epistola 108, la bella lettera delle rimembranze ${ }^{5}$ in cui Seneca traccia anzitutto un vivido ricordo della sua esperienza alla scuola dei Sestii e presso lo stoico Attalo, e poi affronta alcuni problemi di critica letteraria e di interpretazione dei testi in rapporto al magistero filosofico. Seneca dunque, per discutere di questioni critico-letterarie, sceglie di conferire l'aspetto di 'literary memoirs' a due epistole in cui si menzionano scuole e maestri aventi un rapporto diretto, nel bene o nel male, proprio con Seneca padre: Fabiano (nella 100) e la scuola dei Sestii (nella 108), da cui il giovane Seneca, come egli stesso ricorda, fu strappato dalla volontà del severo genitore. ${ }^{6}$

Venendo ora al ritratto letterario di Fabiano, già molti studiosi hanno scrupolosamente annotato le riprese puntuali, da parte di Seneca filosofo, della praefatio

\footnotetext{
4 Penso naturalmente alle epistole 40 e 114, basate sul medesimo principio della corrispondenza tra lo stile e l'animus, tra il modo di vivere e il modo di esprimersi (cf. epist. 114.1: talis hominibus fuit oratio qualis vita): un principio al quale Seneca, forse più di ogni altro scrittore antico, conferì molta importanza e rielaborò in maniera originale, arrivando a sfiorare l'idea (alla quale non fu estraneo, peraltro, l'influsso del padre) che lo stile di ogni scrittore fosse qualcosa di irripetibile e dotato di leggi sue proprie. Sul tema, assai dibattuto, risultano fondamentali gli studi di Setaioli (2000) 111-217 e Berti (2018).

5 Sulla caratura autobiografica della lettera 108 rimando a quanto scriveva Lana (1955) 53: "Questo ricordo carico di affetto sincero, che riappare vivo solo quando Seneca è - e sa di essere - sulla soglia della morte, significa ritorno alle origini, agli ideali di allora. Perché non si tratta di una menzione frettolosa e generica, come a tutti avviene di fare quando il discorso incidentalmente cade su persone accanto a cui si sia vissuti tanti e tanti anni addietro: ma è sentimento di vita vissuta, è nostalgia di beni perduti, è fedeltà riaffiorante [...] a regola di vita troppo a lungo negletta: è insomma la riscoperta delle proprie origini, il ritrovamento della fede di un tempo nel cammino a ritroso sulle vie della memoria".
}

6 Sen. epist. 108.22. 
paterna. ${ }^{7}$ Da parte mia, come già accennato, vorrei affrontare due questioni che, a quanto mi risulta, non hanno finora ottenuto sufficiente attenzione. In primo luogo, partendo dal tema dell'epistola 100, incentrata su un aspetto tecnico dello stile di Fabiano, cioè la sua compositio ${ }^{8}$ tenterò di dimostrare che nel giudizio senecano emerge in filigrana un tratto peculiare dello stile storico, rintracciabile nella tradizione retorica antica. Nel costruire il ritratto di Fabiano come modello di stile filosofico, Seneca sembra alludere alla scrittura storiografica e quasi invitare il lettore a riflettere su tale sovrapposizione, proponendo un insolito quartetto di filosofi (Cicerone, Asinio Pollione, Livio e Fabiano stesso), che forse si potrebbe spiegare, una volta ancora, come un celato riferimento a Seneca padre. In secondo luogo, partendo da alcune corrispondenze strutturali tra il ritratto di Fabiano in Seneca padre e quello presente in Seneca filosofo, proverò a evidenziare il significato di 'icona culturale' che il secondo conferisce a Fabiano, recependo una feconda intuizione paterna ma sviluppandola in maniera autonoma.

\section{Tra filosofia e storiografia: la mollis compositio di Fabiano}

Nel dibattito tra Lucilio e Seneca sullo stile di Fabiano, così come si articola nell'epistola 100, mi sembra di poter cogliere traccia di un'antica riflessione stilistico-retorica sulla compositio, di cui la più cospicua testimonianza si legge nel

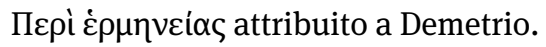

Con un'avvertenza preliminare: non si intende qui stabilire alcun tipo di rapporto diretto fra il trattato di Demetrio e Seneca né tantomeno ipotizzare una dipendenza del secondo dal primo, dal momento che lo vietano sia la natura del

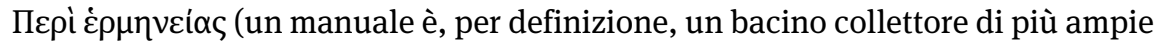
e composite tradizioni confluite nella pratica didattica) sia soprattutto le forti e persistenti incertezze di attribuzione e di datazione sussistenti al suo riguardo. Piuttosto, considerata anche la ricca polifonia che caratterizza la riflessione senecana sullo stile, ${ }^{9}$ si intende suggerire la possibilità di una convergenza, magari

7 Limitandomi ai contributi recenti, segnalo in particolare Cizek (2002), Laudizi (2005), Garbarino (2006), Berti (2018) 211-224; 303-397.

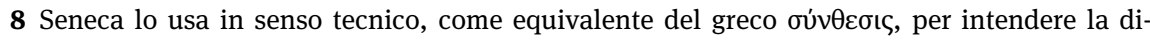
sposizione delle parole nel periodo tanto sotto l'aspetto sintattico quanto rispetto alle qualità ritmiche ed eufoniche: cf. anche epist. 114.15-16, su cui Berti (2018) 151-162.

9 La cui matrice polivalente è magistralmente ricostruita da Setaioli (2000), in particolare 126197. 
tramite un serbatoio comune di fonti e di tradizioni di scuola, con alcune specifiche teorie attestate da Demetrio. ${ }^{10}$

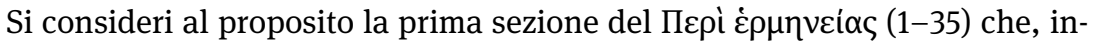
sieme a un passo del terzo libro della Retorica aristotelica ${ }^{11}$ costituiscono la testimonianza più cospicua per ricostruire nelle sue linee principali la teoria del

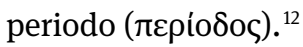

In Demetrio il periodo, inteso come unione di cola e di commata e caratterizzato da una struttura perfettamente circolare, ${ }^{13}$ viene strettamente connesso al

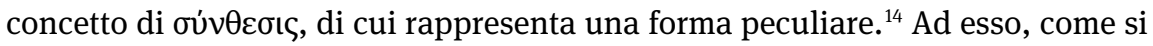
è accennato, è specificamente dedicata la parte introduttiva (1-35, in particolare 10-22), seguita, nel resto dell'opera, dalla più ampia trattazione dei quattro stili e relative degenerazioni (stile grande: 36-127; stile elegante: 128-189; stile semplice: 190-239; stile veemente: 240-304), di ciascuno dei quali si analizzano, in

10 Un'esauriente e documentata discussione sull'attribuzione e la datazione del trattato si può leggere in Chiron (1993) XI-XL. L'ipotesi qui suggerita, se pur con molte cautele, è che esso sia opera di Demetrio di Siria (identificabile con il Demetrio d'Alessandria citato in Diogene Laerzio come ottavo nella lista degli omonimi di Demetrio Falereo) e risalga dunque alla fine del II a.C. o all'inizio del I a.C. L'autore, formatosi ad Alessandria, avrebbe lavorato ad Atene su Aristotele e Teofrasto grazie ai testi confluiti nella biblioteca di Apellicone di Teo e lì avrebbe insegnato retorica fino ad età avanzata, avendo tra i suoi allievi anche il giovane Cicerone. Tale ipotesi, come anche la valorizzazione (ibidem) della presenza di un'influenza stoica nel trattato (in particolare, di teorie stilistiche riconducibili a Panezio), accanto alla matrice aristotelica e peripatetica, sono compatibili con la possibilità di una convergenza con Seneca, tramite un serbatoio comune di fonti e di tradizioni scolastiche.

11 Arist. $r$. 3.9.1409a-1410b.

12 Per il complesso rapporto con Aristotele, che non si limita alla massiccia influenza dello Sta-

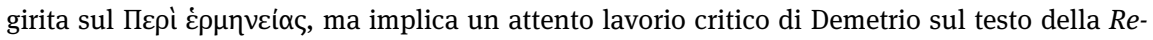
torica, percepibile soprattutto nell'introduzione (1-35) e volto a emancipare le teorie stilistiche dal dominio filosofico, si rimanda ancora all'esauriente discussione in Chiron (1993) LXVIILXXVIII.

13 Il periodo viene paragonato alla pista di un circo, che può essere abbracciata dallo sguardo in qualsiasi momento del percorso, mostrando fin dall'inizio il suo punto di svolta e l'ultima

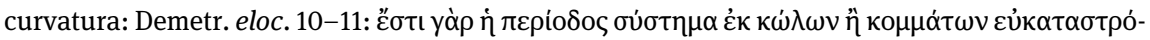

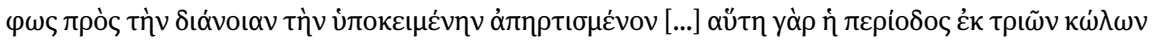

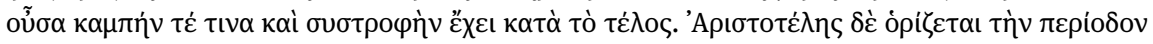

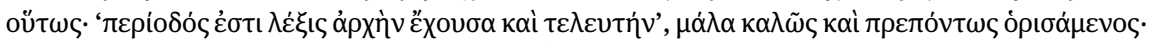

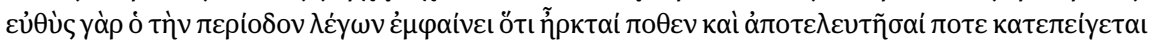

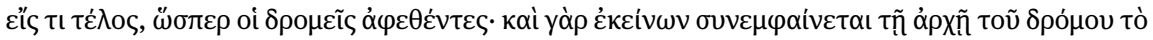

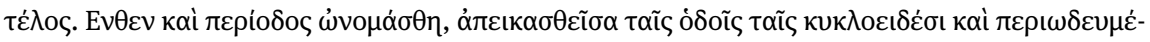
vaıs. Sull'originalità di questa immagine e il suo ruolo determinante nel superamento della teoria aristotelica del periodo cf. Chiron (1993) LXIX-LXX.

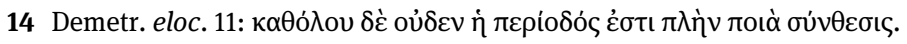




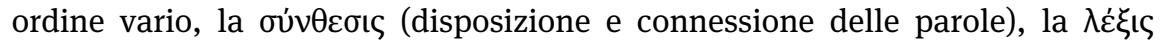

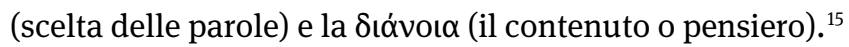

Uno schema analogo, ma abbreviato e in miniatura, mi sembra potersi rintracciare anche nell'epistola 100: nella parte introduttiva (1-2) trovano posto alcune considerazioni sul tipo di periodo che contraddistingue la prosa di Fabiano; nel prosieguo della lettera, l'analisi si amplia al suo stile (oratio), considerato dapprima nell'insieme (3-4), quindi nella scelta delle parole e dei pensieri (5) e poi, più distesamente, negli aspetti della compositio (6-10), riprendendo alcuni punti già esposti nei paragrafi introduttivi e svolgendo un confronto con altri autori. ${ }^{16}$

Rileggere i primi paragrafi dell'epistola senecana alla luce della parte iniziale

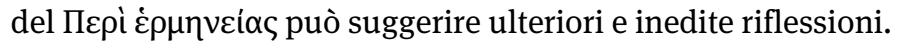

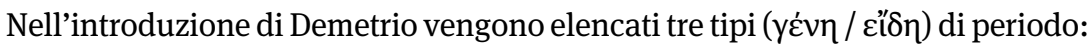

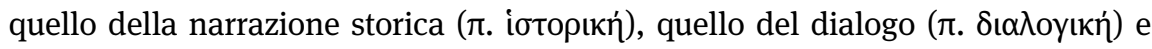

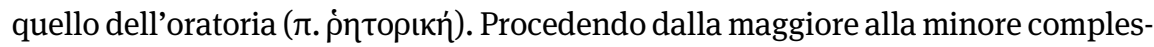
sità del periodo, la trattazione può essere così sintetizzata: la forma del periodare oratorio è concentrica e circolare e richiede una pronuncia rotonda e una mano che batta il tempo. Il periodare della storia non deve essere né tanto allentato (ávet-

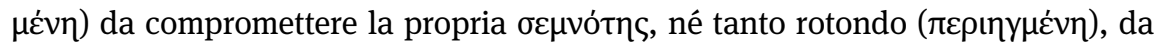
sostituire al ritmo dei fatti esposti il ritmo artificiale di una eccessiva circolarità. Il periodare del dialogo è ancora più allentato e più semplice del periodare della narrazione storica, e poiché i suoi cola sono per così dire 'aggettati' l'uno all'altro, soltanto in chiusura (e quasi a stento) esso si lascia percepire come un periodo in senso proprio; il periodo dialogico, insomma, è definito come una sorta di inter-

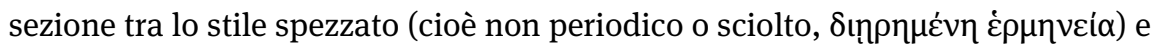

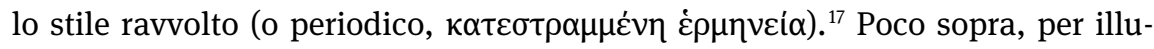
strare la differenza tra questi due fondamentali tipi di stile, Demetrio era ricorso

15 Sulla struttura dell'esposizione dei quattro stili in Demetrio e sulla genesi complessa di questa teoria (che non ha un preciso corrispondente nella retorica antica) cf. Chiron (1993) XLILXI.

16 Chiudono l'epistola due paragrafi (11-12) che tornano a svolgere considerazioni di insieme sullo stile di Fabiano, la sua funzione rispetto al magistero filosofico e il rapporto tra oralità e scrittura.

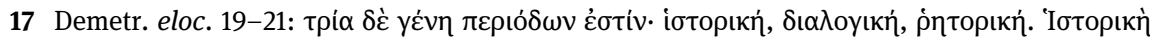

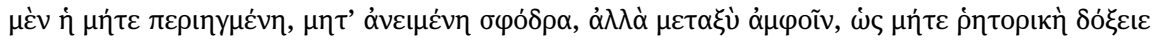

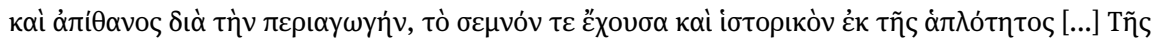

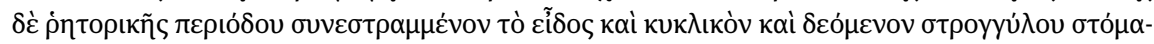

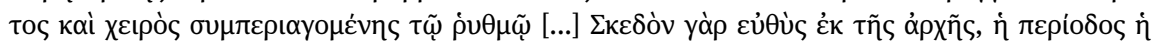

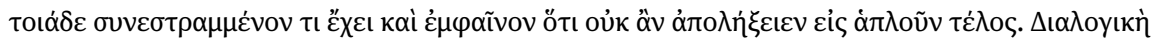


(secondo un uso tradizionale nell'ambito della critica letteraria) ${ }^{18}$ a un paragone architettonico: i cola dello stile periodico assomigliano alle pietre che puntellano e tengono uniti i tetti sferici, ${ }^{19}$ i cola dello stile sciolto assomigliano a pietre che siano state solo gettate, piuttosto che ben collocate, l'una vicina all'altra. ${ }^{20}$

Tornando ora all'epistola 100, il giudizio espresso da Lucilio nei confronti della compositio di Fabiano sembra costruito in modo tale da suggerire un accostamento allo stile periodico del dialogo, tendente decisamente verso uno stile 'sciolto'. Lucilio ritiene infatti che nella compositio di Fabiano le parole siano gettate via, e non siano 'ben conficcate' nell'insieme della frase (effundi verba, non figi): l'immagine, qui adombrata, mi sembra non lontana da quella delle pietre, a copertura delle volte, presente in Demetrio. ${ }^{21}$

Seneca, dal canto suo, non ritiene la compositio di Fabiano così allentata, come vuole Lucilio (Fabianus mihi non effundere videtur orationem sed fundere) né la considera priva di un suo ritmo e di uno sviluppo ampio e continuato (adeo larga est et sine perturbatione, non si cursu tamen veniens); ma, d'altra parte, afferma che essa non è modellata né eccessivamente ripiegata in circolo come vuole il periodare oratorio (illud plane fatetur et praefert, non esse tractatam nec

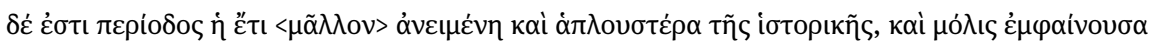

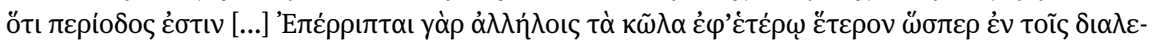

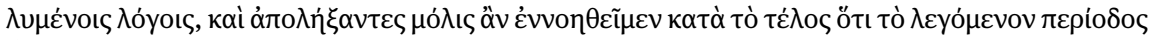

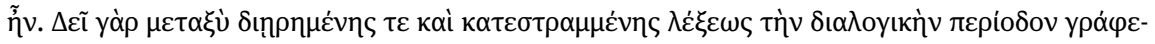

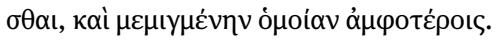

18 Chiron (1993) 7 (cf. anche Demetr. eloc. 14, 33, 108). Il paragone con le pietre si ritrova anche in D.H. 22.148 (II 96, 15 U.-R.).

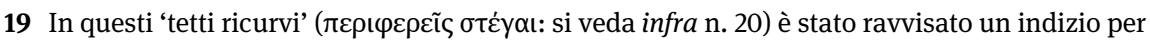
la datazione del trattato di Demetrio al I a.C.: l'aggettivo potrebbe infatti avere un valore tecnico e alludere ai tetti a cupola, conosciuti in Occidente solo a partire da questa data: Lombardo (1999) 97 n. 44 (citando K. Paffenroth).

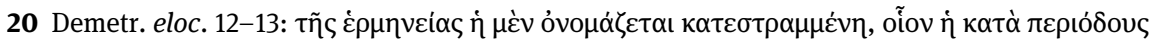

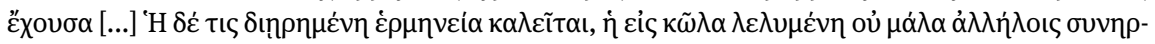

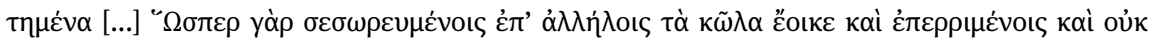

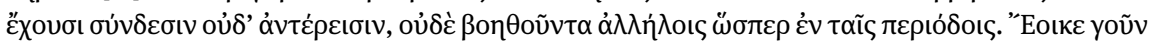

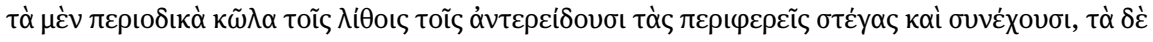

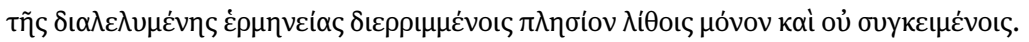

21 Sen. epist. 100.1: [...] compositionem eius accusas. Puta esse quod dicis et effundi verba non figi. La metafora architettonica torna anche in seguito, quando Seneca paragona la compositio di Fabiano a una domus ben fondata ma priva delle raffinatezze e dei lussi all'ultima moda. 
diu tortam). ${ }^{22}$ La posizione mediana che Seneca riconosce alla compositio di Fabiano è dunque affine a quella che Demetrio assegna al periodare di tipo storico, equidistante sia dalla rotondità del periodo oratorio sia dalla scarsa coesione di quello dialogico.

Un riscontro in tal senso è fornito da un passo del De oratore, che chiude la sezione del secondo libro del dialogo dedicata da Cicerone al rapporto tra storiografia e oratoria: la descrizione, per bocca di Antonio, delle principali caratteristiche dello stile storico (non limitate però alla compositio) presenta una significativa corrispondenza con alcuni passaggi dell'epistola 100 riferiti allo stile di Fabiano: anche in Cicerone lo stile della storiografia si caratterizza per la sua ampiezza e fluidità (genus orationis fusum atque tractum) e scorre regolare senza andare a scapito della sua scorrevolezza (cum levitate quadam aequabiliter profluens). ${ }^{23}$

Ci si può domandare, a questo punto, la ragione di questa sovrapposizione del periodare di Fabiano alla compositio di tipo storico. Una prima considerazione è che Seneca possa aver recepito le importanti indicazioni ciceroniane, contenute in un luogo dell'Orator, ${ }^{24}$ secondo cui lo stile del filosofo, nettamente distinto da quello dell'oratore, va accostato piuttosto a quello di altre categorie di scrittori, come i sofisti (cioè gli oratori del genere epidittico), i poeti e, appunto, gli storici. Un tratto comune a tutti questi generi è una oratio più distesa, priva della tensione e dell'energia dell'oratoria; ma in particolare, tra le caratteristiche dello stile storiografico, ${ }^{25}$ Cicerone annovera in tale contesto l'ampiezza e la fluidità (tracta et fluens), già menzionate (sempre a proposito della scrittura storica)

22 Sen. epist. 100.2: Fabianus mihi non effundere videtur orationem sed fundere; adeo larga est et sine perturbatione, non sine cursu tamen veniens. Illud plane fatetur et praefert, non esse tractatam nec diu tortam. Anche i due verbi fatetur e praefert mostrano una significativa convergenza con quanto Demetrio (eloc. 11) afferma sulla intrinseca natura del periodo: un periodo è tale se mostra fin dall'inizio il suo punto di svolta e l'ultima curvatura.

23 Cic. de orat. 2.64: verborum autem ratio et genus orationis fusum atque tractum et cum levitate quadam aequabiliter profluens sine hac iudiciali asperitate et sine sententiarum forensibus aculeis persequendum est. Il passo si può confrontare con Sen. epist. 100.2 e, inoltre, 100.8: non sunt enim illa humilia sed placida et ad animi tenorem quietum compositumque formata, nec depressa sed plana. Deest illis oratorius vigor stimulique quas quaeris et subiti ictus sententiarum. Sull'accezione retorica di fundo e relativi luoghi paralleli: Berti (2018) 320.

24 Cic. orat. 62-64.

25 Cic. orat. 66: huic generi historia finitima est, in qua et narratur ornate et regio saepe aut pugna describitur; interponuntur etiam contiones et hortationes, sed in his tracta quaedam et fluens expetitur, non haec contorta et acris oratio. 
nel passo del De oratore sopra citato, ${ }^{26}$ e aggiunge che esso non ha un andamento ‘curvato' o 'ritorto': proprio l'aggettivo contortus (il cui esatto valore in questo contesto è oggetto di discussione tra gli interpreti) ${ }^{27}$ a mio parere potrebbe serbare una (più tecnica) allusione alla struttura circolare e concentrica del periodare oratorio, segnato da una accentuata circonduzione ( $\pi \varepsilon \rho \propto \alpha ү \omega y \eta ́)$ alla quale, viceversa, il periodare storico deve rinunciare, per non sostituire al ritmo dei fatti esposti il ritmo artificiale di una pronunciata circolarità. ${ }^{28}$ Se tale lettura fosse corretta, la convergenza con quanto nell'epistola 100 si dice della compositio di Fabiano (non esse tractatam nec diu tortam) risulterebbe ancora più pregnante, saldando più tenacemente la compositio del filosofo alla compositio dello storico, secondo un'unica linea interpretativa da Cicerone a Seneca.

La seconda considerazione riguarda i modelli che Seneca, nell'epistola 100, adduce a sostegno del fatto che sulla compositio non esiste una vera e propria regola ${ }^{29}$ e con i quali mette a confronto l'oratio di Fabiano: Seneca intende così dimostrare a Lucilio che questi, pur inferiore ai grandi nomi citati, viene subito dopo di loro ed è pertanto uno scrittore di tutto rispetto. Seneca ci offre dunque una sorta di 'quadriga' dei migliori scrittori latini di filosofia ${ }^{30}$ composta da Cicerone, Asinio Pollione, Tito Livio e appunto Fabiano. Tale quartetto risulta alquanto singolare poiché di Asinio e Livio non sono altrimenti noti scritti filosofici e la notizia senecana è stata pertanto variamente interpretata. ${ }^{31}$

Non è mia intenzione discutere ora tale questione, che peraltro, allo stato attuale delle ricerche, ritengo destinata a rimanere irrisolta. Mi limito pertanto a

26 Queste caratteristiche denotano anche la compositio di Fabiano, secondo il giudizio di Seneca.

27 Berti (2018) 321 (più spesso viene inteso nel senso di 'vibrato', con un'immagine che richiama il lancio del giavellotto).

28 Demetr. eloc. 19-20.

29 Un principio, peraltro, che egli condivide con Seneca padre (cf. contr. 9.6.11: tantus autem error est in omnibus quidem studiis, maxime in eloquentia, cuius regula incerta est, ut vitia quidam sua et intelligant et ament): Setaioli (2000) 162-168; Berti (2007) 183-186.

30 Sul problema della definizione antica di un canone di filosofi romani si veda Berti (2018) 355356.

31 Se Garbarino (2003), sulla base dell'epistola 100, include anche Pollione e Livio nella raccolta di filosofi romani di cui restano frammenti o testimonia, altri studiosi pensano piuttosto che i nomi fatti da Seneca siano chiamati in causa come prosatori in generale o come rappresentanti di tipi diversi di compositio o di differenti maniere stilistiche; una soluzione di compromesso, fondata sul titolo dell'opera di Fabiano citato nell'epistola 100 (Civilium libri, equivalente

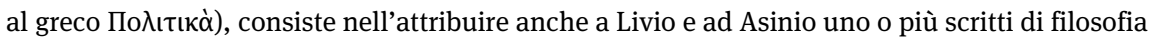
politica, dove fosse concesso ampio spazio alla trattazione storica. Per una sintesi della questione si veda Berti (2018) 355-360. 
qualche osservazione in linea con il tema che stiamo sviluppando: anzitutto, la proposta di una quadriga nell'ambito di una discussione stilistico-letteraria è di per sé un omaggio alla maniera dei 'literary memoirs' di Seneca padre; ${ }^{32}$ in secondo luogo, Asinio e Livio furono due celebri storiografi e Cicerone era altresì famoso per aver fornito contributi teorici di grande importanza sulla scrittura della storia (anzi, nel De legibus, come è noto, egli veniva investito da Attico del ruolo di fondatore latino del genere storiografico, pur in absentia di opera); ${ }^{33}$ non solo, ma Cicerone, Asinio e Livio furono anche declamatori, come ci ricorda Seneca padre nelle Controversiae; infine, nella sesta Suasoria, dedicata alla morte di Cicerone, Asinio e Livio risultano rispettivamente il primo e il secondo degli autori i cui brani, dopo una serie di declamatori, vengono citati da Seneca per fornire ai figli un esempio di scrittura più solida e veritiera (pur sapendo che questi preferiscono di gran lunga i declamatori agli storici). ${ }^{34}$

Concludendo la prima parte del mio contributo e ricapitolando il ragionamento fin qui svolto, ritengo di poter affermare che Seneca nell'epistola 100 abbia voluto affrontare la questione del rapporto tra retorica e filosofia con sguardo retrospettivo, optando per un recupero, nel nome di Fabiano, della scrittura memorialistica del genitore, a metà tra autobiografia e critica letteraria; e abbia inteso saldare insieme, secondo una linea già ciceroniana, lo stile della filosofia e lo stile della storiografia. Tale dibattutissima questione viene qui affrontata da Seneca sul versante tecnico della compositio: egli situa quella di Fabiano in una posizione mediana, affine a quella del periodo storico, a metà tra il periodo oratorio e l'andamento dialogico. A proposito di questa sorta di triangolazione tra compositio storica, filosofica e dialogica, non sarà forse un caso che, di Tito Livio, Seneca citi due tipi di opere, quelle a contenuto espressamente filosofico e i dialoghi, definiti come una forma ibrida tra storia e filosofia. ${ }^{35}$

In tale contesto, Seneca sembra inoltre recuperare allusivamente, attraverso la menzione di Cicerone, Asinio e Livio, il tema del rapporto tra la declamazione

32 Alludo al tetradeum dei migliori declamatori in Sen. contr. 10 praef. 12 (Latrone, Fusco, Albucio, Gallione).

33 Cic. leg. 1.5-9. Sul tema del rapporto di Cicerone con la storiografia, di portata troppo vasta da esaurire in una nota, mi limito a segnalare Leeman (1974) 221-230; Cape (1997) 211-228; Marchese (2011) 152-162.

34 Sen. suas. 6.16: nolo autem vos, iuvenes mei, contristari, quod a declamatoribus ad historicos transeo. Satisfaciam vobis, et fortasse efficiam, ut his sententiis lectis solida et verum habentia recipiatis. Et quia hoc statim recta via consequi non potero, decipere vos cogar, velut salutarem daturus pueris potionem, summa parte poculi.

35 Sen. epist. 100.9: nomina adhuc T. Livium; scripsit enim et dialogos, quos non magis philosophiae adnumerare possis quam historiae, et ex professo philosophiam continentis libros. 
e la scrittura storiografica (e quello, ad esso connesso, della nuova gerarchia dei generi letterari), già affrontato da Seneca padre nella sesta Suasoria. ${ }^{36}$ Seneca figlio rivisita e aggiorna questo tema, piegandolo a esprimere il problema del rapporto tra retorica e filosofia, che del resto, proprio a partire dagli anni della sua formazione, con la comparsa di filosofi professionisti in latino da una parte, e il successo della declamazione dall'altro, aveva assunto una nuova e aggiornata facies. ${ }^{37}$

\section{Sulle orme del padre (II): il ritratto letterario di Fabiano}

Passando ora a sviluppare il secondo punto dell'analisi, proverò a evidenziare il rapporto strutturale intercorrente tra i ritratti letterari di Fabiano, presenti nei due Seneca, e a riflettere sul significato che tale rapporto riveste per l'interpretazione dell'epistola 100.

Entrambi i ritratti presentano una struttura a dittico: Seneca padre delinea (positivamente) lo stile del declamatore mediante il procedimento della бúyкрıбıৎ con lo stile del maestro, Arellio Fusco, di cui si evidenziano soprattutto i difetti; in Seneca figlio lo stile di Fabiano viene descritto mediante il confronto tra il punto di vista di Lucilio (negativo) e quello di Seneca (positivo). Per ciascuno dei due ritratti, dunque, si configurano quattro 'tipi' stilistici, accoppiati a due a due (positivo vs negativo; positivo vs negativo). La caratterizzazione dei quattro tipi avviene secondo un certo numero di tratti ricorrenti, $i$ quali, al di là della generica convenzionalità del linguaggio critico letterario cui appartengono, nell'uno o nell'altro autore si compongono variamente e, talvolta, sono cambiati di segno. È su questa dinamica combinatoria, giocata su corrispondenze o scarti, che vale la pena di riflettere, senza poter qui entrare nel merito della ricostruzione e della valutazione di tutti i tratti e delle varie sfumature dello stile di Fusco e di Fabiano. ${ }^{38}$

36 Berti (2007) 220-222.

37 In sintesi, sui rapporti tra declamazione e filosofia (partendo dal caso di Papirio Fabiano) rimando a Guérin (2012-2013) 21-43 (si veda anche qui infra n. 52); Del Giovane (2015) 175 n.554 (con ulteriori riferimenti bibliografici); Torre (2017) 102-107.

38 Per cui rimando a Berti (2007) 30-31; 67; 180 n.1; 205-206; 273-278; su Fabiano, in particolare, cf. Berti (2018) 221-223; 314-380. 
La бúyкрıбıৎ presente nel ritratto di Seneca padre può essere così schematizzata:

Tab. 5: Confronto tra Fusco e Fabiano: Sen. contr. 2 praef.

\begin{tabular}{|c|c|c|}
\hline & \multicolumn{2}{|c|}{ Seneca padre } \\
\hline & Arellio Fusco & Fabiano \\
\hline \multirow[t]{3}{*}{ Continuità } & Obscuritas $^{\mathrm{a}}(-)$ & Obscuritas $^{\mathrm{a}}(-)$ \\
\hline & Mancanza di vigore oratorio ${ }^{\mathrm{b}}(-)$ & Mancanza di vigore oratorio ${ }^{\mathrm{b}}(-)$ \\
\hline & Ricchezza delle descrizioni ${ }^{c}(+)$ & Ricchezza delle descrizionic $(+)$ \\
\hline \multirow[t]{2}{*}{ Discontinuità } & Mollezza della compositio ${ }^{d}(-)$ & $\begin{array}{l}\text { Fortezza dell'animus / dei praecepta } \\
(+)\end{array}$ \\
\hline & Cultus nimius, luxuria ${ }^{\mathrm{e}}(-)$ & $\begin{array}{l}\text { simplicissima facultas dicendi, non ela- } \\
\text { borata oratio, voluntarius splendor }^{\mathrm{e}}(+)\end{array}$ \\
\hline
\end{tabular}

Come risulta dalla tabella, ${ }^{39}$ in Seneca padre vige anzitutto una continuità tra Fusco e Fabiano per quanto riguarda tre tratti: due di essi vengono considerati difetti (la obscuritas e la mancanza di vigore oratorio), il terzo viene giudicato come un pregio (l'eccellenza e la ricchezza delle descriptiones). Fusco e Fabiano presentano invece una discontinuità per quanto riguarda due difetti del maestro, che l'allievo ha ripudiato: anzitutto, la mollezza della compositio, che Fabiano rifiuta in quanto incompatibile con la forza e l'austerità dei precetti filosofici a cui si è convertito (compositio verborum mollior quam ut illam tam sanctis fortibusque

39 (N.B.: le lettere riprendono quelle in tabella poste in apice) a) contr. 2 praef. 2: $[\mathrm{Fa}]<[\mathrm{Fu}]$ obscuritatem non potuit evadere, haec illum usque in philosophiam prosecuta est; saepe minus quam audienti satis est eloquitur [...] antiquorum [...] vitiorum remanent vestigia. b) contr. 2 praef. 1: [Fu] nihil acre; contr. 2 praef. 2: [Fa] et quotiens inciderat aliqua materia, quae convicium saeculi reciperet, insipirabat magno magis quam acri animo. deerat illi oratorium robur et ille pugnatorius mucro. c) contr. 2 praef. 1: [Fu] nimia licentia vaga et effusa [...] in descriptionibus extra legem omnibus verbis, dummodo niterent, permissa libertas; contr. 2 praef. 3: [Fa] suasoriis aptior erat: locorum habitus fluminumque decursus et urbium situs moresque populorum nemo descripsit abundatius. Numquam inopia verbi substitit, sed velocissimo ac facillimo cursu omnes res beata circumfluebat oratio. d) contr. 2 praef. 1: [Fu] Fusci Arelli [...] compositio verborum mollior quam [Fa] ut illam tam sanctis fortibusque praeceptis praeparans se animus pati posset. e) contr. 2 praef. 1: [Fu]: erat explicatio Fusci Arelli splendida quidem se operosa et implicata, cultus nimis adquisitus [...] splendida oratio et magis lasciva quam laeta; contr. 2 praef. 2: [Fa]: in summa eius ac simplicissima facultate dicendi [...] splendor vero velut voluntarius non elaboratae orationi aderat. 
praeceptis praeparans se animus pati posset); in secondo luogo, la luxuria espressiva di Fusco (erat explicatio Fusci Arelli splendida quidem se operosa et implicata, cultus nimis adquisitus [...] splendida oratio et magis lasciva), che Fabiano abbandona in nome di una simplicissima facultas dicendi e di una non elaborata oratio, cui si accompagna una sorta di spontanea brillantezza (splendor vero velut voluntarius non elaboratae orationi aderat).

Azzardando una prima conclusione, si può affermare che in Seneca padre lo stile di Fabiano ricalchi il paradigma tradizionale, senz'altro ciceroniano nella sua formulazione, del sermo filosofico: uno stile sprovvisto della potenza offensiva e della carica dello stile oratorio, ma austero e del tutto coerente sia con la funzione del docere e del probare che con la dignità dei precetti filosofici e, cosa ancora più importante, perfettamente adeguato alla virtù interiore di un animo sereno, composto e padrone di sé. Particolarmente cogente risulta, pertanto, il confronto con il 'manifesto' dell'Orator, già sopra menzionato. ${ }^{40}$ Seneca padre sembra aver selezionato alcuni tratti della descrizione ciceroniana dello stile filosofico per attribuirli a Fabiano: nello specifico, la mancanza di vigor oratorius; la compostezza che nasce dal dominio delle passioni, quelle proprie e di un pubblico di dotti (in Cicerone: loquuntur cum doctis, quorum sedare animos malunt quam incitare); la 'scelta di castità' (in Cicerone: casta, verecunda, virgo incorrupta quodam modo), rispetto alle lusinghe e agli adescamenti della splendida e luxuriosa oratio. In Fabiano rimangono tutt'al più, come (piccoli) difetti congeniti, alcuni tratti derivati dal tirocinio presso uno dei migliori declamatori del tempo, il cui stile asiano esercitava un indubbio fascino tra gli scholastici e il loro pubblico più giovane ma al quale, peraltro, Seneca padre guarda con una certa indulgenza, pur riconoscendone la natura viziosa. ${ }^{41}$

40 Cic. orat. 62-64: quamquam enim et philosophi quidam ornate locuti sunt, si quidem Theophrastus divinitate loquendi nomen invenit et Aristoteles Isocratem ipsum lacessivit et Xenophontis voce Musas quasi locutas ferunt et longe omnium quicumque scripserunt aut locuti sunt exstitit<et suavitate $>$ et gravitate princeps Plato - tamen horum oratio neque nervos neque aculeos oratorios ac forenses habet. Loquuntur cum doctis, quorum sedare animos malunt quam incitare, et de rebus placatis ac minime turbulentis docendi causa non capiendi loquuntur, ut in eo ipso, quod delectationem aliquam dicendo aucupentur, plus nonnullis quam necesse sit facere videantur. Ergo ab hoc genere non difficile est hanc eloquentiam de qua nunc agitur secernere. Mollis est enim oratio philosophorum et umbratilis nec sententiis nec verbis instructa popularibus nec vincta numeris, sed soluta liberius; nihil iratum habet, nihil invidum, nihil atrox, nihil miserabile, nihil astutum; casta, verecunda, virgo incorrupta quodam modo. Itaque sermo potius quam oratio dicitur. Quamquam enim omnis locutio oratio est, tamen unius oratoris locutio hoc proprio signata nomine est.

41 Berti (2007) 205-206. 
Se ora passiamo a considerare l'epistola 100, noteremo che sia Lucilio sia Seneca ripropongono per Fabiano tratti di continuità o di discontinuità rispetto al giudizio di Seneca padre, ma li valutano in modo antitetico tra loro e, talora, anche rispetto al modello.

Tab. 6: Confronto tra Sen. contr. 2 praef. e Sen. epist. 100

\begin{tabular}{llll}
\hline Seneca padre & & Seneca figlio & \\
\hline Fusco & Fabiano & Fabiano (per Lucilio) & Fabiano (per Seneca) \\
Cultus nimius (-) & non elaborata oratio $(+)$ & $\begin{array}{l}\text { Mancanza di ricerca- } \\
\text { tezza formale }(-)\end{array}$ & $\begin{array}{l}\text { Mancanza di ricerca- } \\
\text { tezza formale }(+)\end{array}$ \\
& Mancanza di oratorium robur & Mancanza di orato- & Mancanza di oratorius \\
& e di pugnatorius mucro $(-) \rightarrow$ & rius vigor $(-)$ & vigor (-) \\
summa inae- & & Mancanza di varietà & \\
$\begin{array}{l}\text { qualitas }(-) \\
\text { Mollis composi- }\end{array}$ & $(-)$ & \\
tio $(-)$ & Mollis compositio $(-)$ & Mollis compositio * \\
\hline
\end{tabular}

Come si evince dalla seconda tabella, le critiche di Lucilio a Fabiano riprendono, e talora manipolano, alcuni tratti della prefazione al secondo libro delle Controversiae: Lucilio ribalta di segno un tratto positivo che Fabiano aveva in Seneca Padre (la mancanza di ricercatezza); ${ }^{42}$ ne recepisce un difetto come tale (la mancanza di vigore oratorio); ${ }^{43} \mathrm{e}$, infine, lamenta in Fabiano l'assenza di un tratto dello stile di Arellio (la summa inaequalitas della sua oratio), che in Seneca padre costituiva un difetto, ma che ora viene invece giudicato positivamente: Lucilio desidererebbe infatti in Fabiano una più varia tavolozza di stili. ${ }^{44}$

42 Sen. epist. 100.5: videbimus quid parum recisum sit, quid parum structum, quid non huius recentis politurae; 100.8: humilia praeterea tibi videri dicis omnia et parum erecta; 100.11: non erunt sine dubio singula circumspecta nec in se collecta.

43 Sen. epist. 100.8: deest illis oratorius vigor stimulique quos quaeris et subiti ictus sententiarum; 100.10: sed non praestat omnia: non est fortis oratio eius [...] non est violenta [...]. 'Desideres' inquis 'contra vitia aliquid aspere dici, contra pericula animose, contra fortunam superbe, contra ambitionem contumeliose. Volo luxuriam obiurgari, libidinem traduci, impotentiam frangi'.

44 Sen. epist. 100.10: sit aliquid oratorie acre, tragice grande, comice exile. Come nota giustamente Berti (2018) 366, in questa frase non si deve cogliere un'allusione alla teoria dei tria genera dicendi, ma piuttosto l'insistenza sulla varietà dei toni dello stille della parenesi filosofica e la proposta di un ideale di stile filosofico "che, in funzione di una maggiore efficacia parenetica, comporta il superamento dei confini tra i tradizionali generi letterari, ma anche tra prosa e poesia”. 
Seneca, da parte sua, controbatte alle critiche di Lucilio recuperando il principale tratto positivo che Fabiano aveva nella seconda prefazione e che, per Seneca padre, rappresentava un tratto di discontinuità rispetto al vitium fondamentale di Arellio, ovvero l'assenza di cultus e di luxuria nell'espressione. ${ }^{45}$ Seneca conclude la lettera affermando che lo stile di Fabiano è nel complesso magnifico anche se non estremamente ricercato e lavorato nei singoli particolari e, talora, sprovvisto di forza. ${ }^{46}$ Come si può vedere, nel controbattere alle critiche di Lucilio, Seneca si rifà al medesimo paradigma ciceroniano utilizzato dal genitore: lo stile di Fabiano, specchio del suo animus compositus, procede in perfetto equilibrio e senza alcuna difformità. ${ }^{47}$ Ma c'è almeno un punto che pare sfuggire alla fitta griglia delle corrispondenze, fin qui stabilita, tra la seconda prefazione alle Controversiae e l'epistola $100 .^{48}$

Dal paradigma ciceroniano proposto nell'Orator, Seneca ha selezionato, attribuendolo allo stile di Fabiano, un tratto che Seneca padre aveva invece obliterato, cioè appunto la mollis compositio. In Cicerone, come già si è accennato, essa rappresenta il marchio distintivo dello stile del filosofo che, coltivato all'ombra della schola, si può definire più come una conversazione (sermo) che come discorso (oratio) in senso proprio. ${ }^{49}$

Nella prefazione al secondo libro delle Controversiae una mollior compositio viene attribuita ad Arellio Fusco e rappresenta proprio quel tratto dello stile del maestro che Fabiano ripudia perché non più adeguato alla costituzione di un animo che si prepara a ricevere i forti precetti filosofici. ${ }^{50}$ Viceversa, nell'epistola 100 un tipo di compositio 'allentata' viene attribuita tout-court a Fabiano da Lucilio ed è il principale oggetto del dibattito tra questi e Seneca; essa non viene mai negata di per sé e, anzi, Seneca finisce per rivalutarla come marca stilistica del

45 Sen. epist. 100.6 (il paragone con la domus): desit sane varietas marmorum et concisura aquarum cubiculis interfluentium et pauperis cella et quidquid aliud luxuria non contenta decore simplici miscet: quod dici solet, domus recta est.

46 Sen. epist. 100.11: non erunt sine dubio singula circumspecta nec in se collecta nec omne verbum excitabit ac punget, fateor; exibunt multa nec ferient et interdum otiosa praeterlabetur oratio, sed multum erit in omnibus lucis, sed ingens sine taedio spatium; 100.12: sine commendatione partium singularium in universum magnificus.

47 Sen. epist. 100.8: non sunt enim illa humilia sed placida et ad animi tenorem quietum compositumque formata, nec depressa sed plana.

48 In Tab. 6 esso è contrassegnato da asterisco.

49 Cic. orat. 64: mollis est enim oratio philosophorum et umbratilis nec sententiis nec verbis instructa popularibus nec vincta numeris, sed soluta liberius [...] Itaque sermo potius quam oratio dicitur.

50 Sen. contr. 2 praef. 1: Fusci Arelli [...] compositio verborum mollior quam ut illam tam sanctis fortibusque praeceptis praeparans se animus pati posset. 
maestro appellandosi, tra l'altro, alla compositio sine infamia mollis di Cicerone, quale autorevolissimo modello che la legittimerebbe. ${ }^{51}$

La domanda che sorge inevitabile a questo punto è perché mai Seneca padre abbia eliminato dallo stile filosofico di Fabiano la mollis compositio, che risulta perciò l'unico (e fondamentale) tratto, tra quelli presenti nel suo modello ciceroniano, a non essere recepito; e perché, viceversa, Seneca figlio la recuperi, come oggetto del dibattito tra lui e Lucilio e, pur in forma indiretta, cioè attraverso il complicato intreccio di obiezioni e contro-obiezioni, la attribuisca a Fabiano come precipua marca di stile filosofico, legittimata dall'esempio di Cicerone e pertinente, come sopra abbiamo visto, allo stile della storiografia.

Credo che una possibile risposta vada cercata nella sempre più accentuata pervasività della retorica nelle varie forme di discorso e di comunicazione intellettuale nella prima età imperiale, e nelle diverse modalità di percepirla e di rappresentarla da parte del padre e del figlio.

Una certa osmosi tra retorica e filosofia viene avvertita come già in atto da Seneca padre. ${ }^{52}$ Nella seconda prefazione Fabiano viene presentato come l'esempio perfetto di tale osmosi: infatti, tentando di convincere il figlio Anneo Mela (cui è

51 Sen. epist. 100.7: lege Ciceronem: compositio eius una est, pedem curvat lenta et sine infamia mollis. Il giudizio sulla compositio di Cicerone corrisponde a epist. 114.16 (quidquid illa in exitu lenta, qualis Ciceronis est, devexa et molliter detinens nec aliter quam solet ad morem suum pedemque respondens?): cf. Berti (2018) 344-345.

52 Sul gioco di influenza reciproca e, insieme, di differenziazione tra le due pratiche concorrenti del discorso, quello declamatorio e letterario da un lato e quello filosofico dall'altro, così come emerge dall'opera di Seneca padre, si rimanda all'analisi di Guérin (2012-2013: 41-42), le cui conclusioni sono sostanzialmente condivisibili: la declamazione, diventata, da semplice intrattenimento che era, una vera e propria esibizione retorica pubblica, inaugura un tipo di eloquenza tutta stilistica, in cui l'inventio, che tradizionalmente costituiva un potenziale spazio di espressione per i saperi intellettuali, perde importanza e diventa del tutto marginale rispetto alla cura esclusiva delle innovazioni formali a discapito dei contenuti; la declamazione orienta in profondità le percezioni e i gusti del pubblico e diventa un quadro estetico di riferimento, capace di indirizzare tutte le forme di discorso: il grado di influenza della declamazione sui vari generi letterari può essere vario, ma la testimonianza di Seneca padre (e di altri autori più tardi) non lascia dubbi sul fatto che nel I d.C. la declamazione pretende di influenzare l'intera produzione in prosa; nasce quindi una forma di concorrenza sociale tra i due mondi intellettuali, quello dei declamatori e quello dei filosofi, in cui la posta in gioco è il favore del pubblico e il successo mondano. Ciò che, dello studio di Guérin, non mi pare condivisibile è la valutazione molto riduttiva del ruolo di Seneca filosofo (con particolare riferimento all'epistola 100) in questo ampio dibattito culturale, come un semplice ritorno a una posizione di rigida separazione tra le due sfere, in linea con la (superata) posizione ciceroniana. Come dirò a breve, il quadro a mio parere è più complesso e Seneca filosofo si rivela molto meno conservatore di quanto ci si possa aspettare. 
dedicata la praefatio) a non trascurare del tutto la pratica della declamazione, pur dopo aver intrapreso gli studi filosofici, Seneca gli indica in Fabiano l'esempio di una perfetta conciliazione tra i due ambiti (facilis ab hac in omnes artes discursus est: instruit etiam quos non sibi exercet). Già declamatore di scuola, quando ormai era diventato un disertore (transfuga), cioè era passato alla scuola sestiana, Fabiano continuava tuttavia a declamare come esercizio propedeutico per la filosofia; e lo faceva così diligentemente che si sarebbe potuto pensare che si esercitasse proprio in vista della declamazione, non della filosofia stessa. ${ }^{53} \mathrm{Se}-$ neca padre, però, rappresenta questa transizione ancora in una forma tradizionale, ammettendo sì la possibilità di un facile discursus, ma attenendosi ancora al paradigma ciceroniano di una corretta e sostanziale separatezza di ambiti. Tale paradigma viene al più aggiornato, sostituendo implicitamente uno dei due ambiti concorrenti (l'oratoria) con un'altra forma retorica più alla moda (la declamazione appunto). In quest'ottica Seneca padre non può dunque fare a meno di eliminare dallo stile di Fabiano il tratto della mollis oratio: infatti, nell'evolversi delle pratiche declamatorie, questo tratto aveva assunto uno spettro di significati e di forme artistiche molto più ampie rispetto al recente passato ed era diventato a tutti gli effetti una caratteristica, se non un vitium dello stile moderno. ${ }^{54}$

53 Sen. contr. 2 praef. passim: cum repeterem, quos umquam bene declamantes audissem, occurrit mihi inter alios Fabianus philosophus, qui adulescens admodum tantae opinionis in declamando, quantae postea in disputando fuit. exercebatur apud Arellium Fuscum [...] Haec eo libentius, Mela, fili carissime, refero, quia video animum tuum a civilibus officiis abhorrentem et ab omni ambitu aversum hoc unum concupiscentem, nihil concupiscere, ut eloquentiae tamen studeas. Facilis ab hac in omnes artes discursus est; instruit etiam quos non sibi exercet. Nec est, quod insidias tibi putes fieri, quasi id agam, ut te bene cedentis studii favor teneat; ego vero non sum bonae mentis impedimentum: perge quo inclinat animus, et paterno contentus ordine subduc fortunae magnam tui partem. [...] Sed proderit tibi in illa, quae tota mente agitas, declamandi exercitatio, sicut Fabiano profuit. Qui aliquando, <cum> Sextium audiret, nihilominus declamitabat et tam diligenter, ut putares illum illi studio parari, non per illud alteri praeparari. [...] Apud Blandum diutius quam apud Fuscum Arellium studuit, sed cum iam transfugisset, eo tempore quo eloquentiae studebat non eloquentiae causa. Cf. Citti (2005) 187-189; Berti (2007) 219-220; Guérin (2012-2013) 21-43.

54 Berti (2018) 105 (a proposito della caratterizzazione di Mecenate come mollis in Sen. epist. 114.7): "D'altra parte mollis è anche un termine della critica letteraria, che analogamente al gr. $\mu \alpha \lambda \alpha \kappa o ́ \varsigma$ si applica a un genus dicendi dolce e gradevole (cf. ad es. Cic. de orat. 2.95; Brut. 38; 132; 274), ma che, volto in malam partem, definisce uno stile languido e snervato, con la solita connotazione di effeminatezza (cf. ad es. Sen. contr. 2 praef. 1; 9.2 .24 [...])”; 345 (a proposito della compositio di Cicerone in Sen. epist. 110.7): "L'accusa di praticare una mollis compositio era stata notoriamente rivolta a Cicerone dai suoi avversarsi atticisti, come attesta Quint. inst. 12.10.12: quem [sc. Ciceronem] tamen et suorum homines temporum incessere audebant ut tumidiorem et Asianum et redundantem [...] et in compositione fractum, exultantem ac paene, quod procul absit, 
A rendere ormai irricevibile, per Seneca padre, la mollis oratio del filosofo ciceroniano può aver contribuito, tra l'altro, l'epiteto umbratilis, ad essa associato nel passo più volte citato dell'Orator. ${ }^{55}$ Già altrove, in Cicerone, tale aggettivo designava i declamatori (che tengono i loro discorsi nel chiuso delle scuole, all'opposto degli oratori, che parlano all'aria aperta del forum) $;{ }^{56}$ a Seneca padre doveva suonare senz'altro come etichetta specifica della schola di retorica, risultando del tutto inutilizzabile per la professione del filosofo.

Una controprova può essere fornita da un altro ritratto di Seneca, quello dedicato ad Albucio Silo nella praefatio al settimo libro: costui è un declamatore che si atteggia a filosofo e che appare come la brutta copia di Fabiano (anzi, un pessimo imitatore in senso proprio, visto che ne aveva frequentato assiduamente le lezioni). ${ }^{57}$ In apparenza, Albucio appare preoccupato di che cosa dire e non di come dirlo ("tanto da poter applicare a se stesso il motto cum rem animus occupavit, verba ambiunt, ovvero acuta variazione del precetto rivolto da Catone al figlio Marco [...] rem tene, verba sequentur"), ${ }^{58}$ ma solo per il timore di sembrare un declamatore in cerca di successo; infarcisce il discorso di concetti filosofici ma in modo intempestivo, sviluppando oltre misura le quaestiones; incostante e mutevole, sollicitus e non affatto securus, ${ }^{59}$ si rivela in pieno uno scholasticus: a un certo punto della sua carriera, dopo essere incorso in uno spiacevole incidente, decide di non parlare più nel foro, giudicando la declamazione l'unica forma in grado di conferire un sicuro successo.

viro molliorem; forse è per questo, oltre che per la connotazione negativa comunemente assunta da mollis come termine della critica letteraria [...] che Seneca, in un contesto in cui non vuole insistere sui difetti della compositio ciceroniana, aggiunge la precisazione sine infamia, così da togliere all'aggettivo ogni risonanza peggiorativa”.

55 Cic. orat. 64: mollis est enim oratio philosophorum et umbratilis.

56 Cic. de orat. 1.157: educenda deinde dictio est ex hac domestica exercitatione et umbratili medium in agmen, in pulverem, in clamorem, in castra atque in aciem forensem; subeundus visus hominum et periclitandae vires ingenii, et illa commentatio inclusa in veritatis lucem proferenda est; sul passo e la sua eredità in Seneca padre cf. Berti (2007) 128-132.

57 Su Albucio in Seneca Padre: Citti (2005) 193-198; Berti (2007) 143-149; Guérin (2012-2013) $28-32$.

58 Citti (2005) 195-196.

59 Sulla contrapposizione tra sollicitus e securus in Seneca padre (contr. 7 praef. 6) e in Seneca filosofo (epist. 100.4) in relazione allo stile come espressione dell'animo, cf. Setaioli (2000) 123124. 
Seneca pare dunque costruire il ritratto, letterario e psicologico, di Albucio in antitesi a quello di Fabiano (al quale peraltro lo accomunano eleganza e brillantezza espressiva); ${ }^{60}$ e, se letta sullo sfondo della seconda, la settima prefazione rivela una chiara percezione della differenza tra declamatori e filosofi e l'intenzione, che mi pare altrettanto netta, di smascherare indebiti tentativi di sconfinamenti o, peggio, di contraffazioni. ${ }^{61}$

Venendo ora all'epistola 100, Seneca recupera allo stile di Fabiano il tratto ciceroniano della mollis compositio già obliterato dal padre; anzi, il recupero avviene nel nome stesso dell'Arpinate, che lo incarnerebbe nella sua prosa. ${ }^{62}$ Per trovare la motivazione di un simile recupero, dobbiamo anzitutto rimarcare che, nell'incipit della lettera, al termine compositio viene attribuita una valenza tecnica in relazione alla teoria retorica del periodo e che, tramite il carattere più allentato del periodare di Fabiano, si stabilisce un'intrinseca affinità (anch'essa, peraltro, riconducibile a una matrice ciceroniana) tra scrittura storiografica e scrittura filosofica.

Ed è appunto il ricorso al medium della storiografia, a mio parere, a garantire che il recupero apparentemente nostalgico della posizione ciceroniana non sia espressione, da parte di Seneca, di un più o meno pronunciato conservatorismo. ${ }^{63}$

60 Sen, contr. 2 praef. 2: splendor vero velut voluntarius non elaboratae orationi aderat (Fabiano); contr. 7 praef. 2: splendor orationis quantus nescio an in ullo alio fuerit.

61 Si leggano al proposito le giuste considerazioni di Guérin (2012-2013) 36-37: "Chez Sénèque le père comme chez Sénèque le jeune, la figure de Papirius Fabianus est utilisée pour décrire cette frontière entre le deux modes d'expression déclamatoire et philosophique. Mais contrairement à Albucius, Fabianus - ancien déclamateur et philosophe reconnu - représente le versant positif de cette rupture en ce qu'il incarne la capacité à manier les deux types de parole, sans pour autant les confondre [...] À partir de ce constat, nous voudrions montrer que Sénèque le père, tout en affirmant l'hétérogenéité stylistique de la philosophie et de la déclamation, parvient à penser une forme d'enrichissement d'une pratique par une autre, mais dans un sens qui n'et pas celui qu'aurait souhaité Cicéron: pour le rhéteur, c'est la déclamation qui rendra le philosophe éloquent, pourvu, une fois encore, qu'il sépare fermement les différents genres de discours qu'il pratique".

62 Sen. epist. 100.7.

63 Come sostengono, pur con varietà di accenti, diversi interpreti, tra cui ad es. Setaioli (2000) 118-122; 160-172 e Guérin (2012-2013), parlando di un allineamento della posizione senecana su quella di Seneca padre o anche di un suo arretramento rispetto al genitore e, sicuramente, rispetto a Lucilio, schierato su una linea più modernista. Più convincente mi pare, al proposito, Garbarino (2006) 69-71, che rivaluta la struttura a dittico nell'epistola e ritiene pertanto la divergenza di opinioni tra Seneca e Lucilio nient'altro che un espediente letterario per esporre in una forma dialetticamente più efficace le idee dell'autore in merito allo stile filosofico. Le due voci dell'epistola, secondo la studiosa, non rispecchiano due diverse posizioni (pro e contro lo stile di Fabiano) ma, piuttosto, due diverse prospettive, espresse su due piani diversi. Lucilio assume 
Al contrario, fatta salva l'assoluta priorità per il filosofo delle res sui verba, Seneca mi pare qui registrare, con lucida consapevolezza, la fluidità dei confini tra declamazione e filosofia, inserendosi a pieno titolo nel dibattito coevo sulla ridefinizione dei generi letterari e seguendo, ancora una volta, per poi superarle, le orme del genitore.

Come infatti Seneca padre, nella sesta Suasoria, proponeva alcuni brani degli storici per competere con i declamatori su un tema alla moda (la morte di Cicerone), garantendo nel contempo la maggiore solidità dei primi sui secondi, così nell'epistola 100 Seneca pare aggiornare sulla falsariga del genitore la discussione sul problema di quale stile per il filosofo, proponendo come modelli un insolito quartetto di filosofi, che erano stati tutti anche declamatori, tre dei quali anche storici e, non da ultimo, protagonisti della sesta Suasoria.

Se letta in tal senso, allora, l'epistola 100 sembra testimoniare molto più la percezione di una osmosi di fatto tra i generi della prosa, con la quale bisogna certamente fare i conti per stabilire delle gerarchie ma da cui non si può affatto prescindere, che un miope arroccamento su posizioni di assoluta (e astratta) separazione tra filosofia e retorica.

\section{Conclusioni}

Dall'analisi dell'epistola 100, che ho proposto in questa sede, il retore e filosofo Fabiano risulta a tutti gli effetti un trait-d'union generazionale, che Seneca eredita dalla scrittura memoriale del padre e utilizza quale strumento per interpretare, anzi rappresentare alcuni tratti significativi della storia della cultura nella prima età imperiale, lasciando per così dire 'a vista' varie tensioni irrisolte che la percorrono. Il movimento retrospettivo, adottato nell'epistola, non mi pare pertanto un passo indietro ma, piuttosto, una voluta strategia per aggiornare un lungo dibattito sullo stile filosofico, alla luce delle contraddizioni a lui contemporanee, nella consapevolezza della veloce evoluzione dei modelli culturali. Se-

il punto di vista del critico letterario e accetta, quale suo presupposto, di applicare i criteri retorici alla formulazione e alla valutazione di uno stile proprio del filosofo; la difesa di Fabiano da parte di Seneca poggia invece su premesse non già retoriche ma filosofiche e proclama il principio della assoluta prevalenza dei contenuti sulla forma. Pur condividendo le premesse di questa interpretazione, credo tuttavia che il gioco delle voci sia ancora più complesso di quello descritto da Garbarino e che la posizione di Seneca, anche come persona loquens nel contesto dell'epistola, non sia riducibile a quella del filosofo attestato su un piano 'altro' rispetto al declamatore e, in questo, riveli il suo tratto più moderno. 
neca ripropone, mutatis mutandis, le dinamiche del dibattito sullo stile storiografico già presente in Cicerone e, in tempi a lui più vicini, in Seneca padre, allo scopo, certamente, di ribadire la supremazia della filosofia sulla declamazione, ma condividendo lo stesso orizzonte di istanze culturali.

Queste istanze e queste contraddizioni, nate in senso alla modernità, in Seneca non vengono mai né celate né semplificate, ma danno vita nel suo epistolario a una posizione estremamente variegata, che coinvolge inevitabilmente anche il tratto antitetico alla mollis compositio, cioè quel vigore espressivo che Lucilio ricerca inutilmente in Fabiano e che viene specificamente connesso all'efficacia del magistero etico perché identificato come lo strumento più idoneo alla parenesi del filosofo. In questo caleidoscopico scenario, che nell'epistola 100 viene espresso mediante l'intreccio delle voci - non solo quelle di Lucilio e di Seneca, ma anche di Seneca padre e di Cicerone -, anche la distinzione e la complementarietà tra sermo (cui pertiene la mollis compositio) e admonitio (cui si connette il vigore espressivo), di antica e illustre tradizione (e altrove adottata, come è noto, da Seneca stesso) ${ }^{64}$ vengono incessantemente rinegoziate e mai definitivamente stabilite.

64 Sen. epist. 38.1-2; Berti (2018) 215-219; Setaioli (2000) 111-141. 
